\title{
Hypothesis Selection in Grammar Acquisition
}

\author{
Masaki KIYONO* and Jun'ichi TSUJII \\ Contre for Computational Linguistics \\ University of Manchester Institute of Science and Technology \\ PO IBox 88, Mauchester M60 1QD \\ United Kingdom \\ kiyono@ccl.umist.ac.uk, tsujii@ccl.umist.ac.uk
}

\begin{abstract}
'This paper presents some techniques for selecting linguistically adequate hypotheses of new grammatical knowlodge to be used as resources of grammatical knowledge acquisition. In our framework of linguistic knowledge accuisition, a rulebased hypothesis generator is invoked in case of parsing failures and all the possible hypotheses of new grammar rules or lexical entries are gonerated from partial parsing results. Although each hypothesis could recover the defects of the existing granmmar, the greater part of hypotheses are linguistically unnatural. The techniques we propose here prevent such unnatural hypotheses from being generated without discarding plausible ones and make the following corpus-based acquisition process nore ellicient and nore reliable.
\end{abstract}

\section{Introduction}

Reusability of existing linguistic knowledge is the most important requirement for the rapid development of practical nalural lauguage proccssing systems. In order to rcalize automatic customization of existing linguistic knowledge to each application domain, we proposed a new approach of linguistic knowledge acquisition, which is a combiuation of symbolic and statistical approaches [Kiyono and Tsujii, 1993].

T'he framework of our approach is shown in ligure 1. The acquisition flow starts with executing the parse of each sentence in a corpus. If parsing failed, the 'Hypothesis Generator' produces the hypotheses of additional grammatical knowledge, each of which could recover the incompleteness of the existing grammar. After itcrating this hypothesis generation process for all the sentences in the corpus, the hypotheses are passed to the statistical analysis process and finally plansible hypotheses are chosen as new knowledge by observing statistical properties of the liypotheses.

Unlike robust parsing [Mcllish, 1989; Gooser, 1992; Douglas and Dale, 1992] or non-statistical approach for grammar acquisition, our approach does not require a mechanism to detect the cause of the parsing failure in the sentencial analysis phase and therefore the 'Iypothesis Generator' may output, all the possible hypotheses. Ilowever, the greater part of hypotheses gencrated by a simple deductive mechanism are unmatural revisions of the existing grammar. F'or example, even a rule which derives a top node category $S$ directly from the input string of words might be hypothesized.

"also a stafl member of Matsushita filectric Ind ustrial Go, Lthl, Shinagawa, Tokyo, JAPAN.

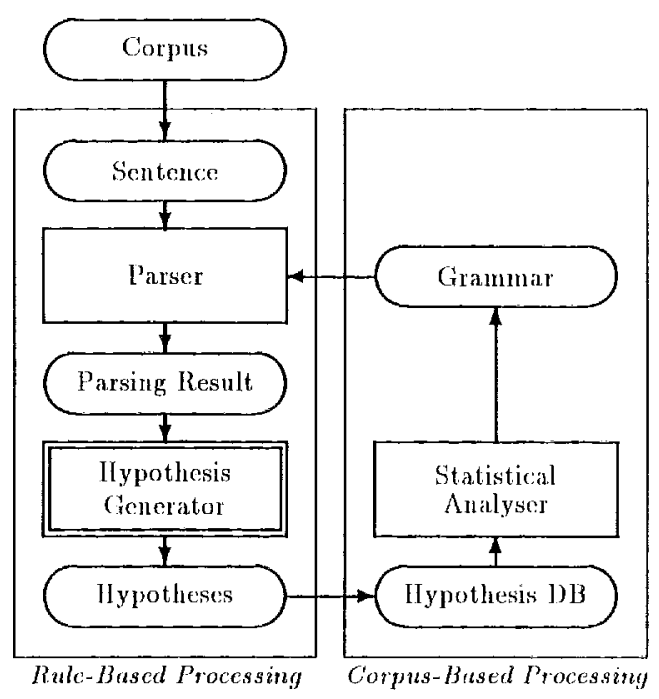

Figure 1: Framework of Grammar Acquisition

Linguistically unnatural hypotheses havo harmful offects on the following corpus-based process, not only making the process inefficient but also in terfering with statistical datia as noise. In this paper, sone tecliniques to remove such inadequate hypotheses are proposed and the results of experiments which show the eflectiveness of the proposed techniques are also discussed.

\section{Grammax Hypothesizing}

\subsection{Grammar Formalism}

'The grammar formalism we use is a conventional unification-based grammar. Fach grammir rule is written in the form of a combination of a context-free rule and feature unification functions. This Tormalism is not specific to any linguistic theory, but we introduced a 11 umber of concepts widely accopted in linguistic theorics, such as grammatical functions, subcalegorization franes, and X-bar theory.

The parsing system we introduced to apply our grammar formalism is a system called SAX [Matsumoto, 1986]. SAX uses the concepts of active and inactive edges of Chart Parsing and analyses an input sentence with a bottom-up and parallel algorithru. As the grammar hypothesizing algorithm is supposed to refer partial parsing results of unsuccessfully parsed sentences, we slightly modified SAX so that it oulputs inactive edges as partial parsing results. 


\subsection{Basic Algorithm}

When SAX fails to parsc a sentence, no inactive edge of category $S$ spanning the whole sentence exists in the parsing result. Grammar hypothesizing is a process to introduce this inactive edge by augmenting the current grammar. The basic part of the hypothesis generation algorithm is written as follows:

[Algorithm] $A n$ inactive edge $\left[i e(A): \boldsymbol{x}_{0}, \boldsymbol{x}_{n}\right]$ can bo introduced from $x_{0}$ to $x_{n}$, with label $A$, by each of the hypotheses generated by the following two steps.

[Step 1] For each sequence of inactive edges, $\left[i e\left(B_{1}\right): x_{0}, x_{1}\right], \ldots,\left[i e\left(B_{n}\right): x_{n \ldots 1}, x_{n}\right]$, spanning from $x_{0}$ to $x_{n}$, generates a new rulc.

$$
A \Rightarrow B_{1}, \cdots, B_{n}
$$

[Step 2] For each existing rule $A \Rightarrow A_{1}, \cdots, A_{n}$, find an incomplete sequence of inactive odges, $\left[i e\left(A_{1}\right): x_{0}, x_{1}\right], \ldots,\left[i e\left(A_{i-1}\right): x_{i-2}, x_{i-1}\right]$, $\left[i e\left(A_{i+1}\right): x_{i}, x_{i+1}\right], \ldots,\left[i e\left(A_{n}\right): x_{n-1}, x_{n}\right]$, and call this algorithm for $\left[\operatorname{ie}\left(A_{i}\right): x_{i-1}, x_{i}\right]$

Feature Structures: A rule generated in [Step 1] cotld be a lexical entry when this top-down algorithm reaches the bottom. As we adopted a unificationbased granmar formalism, we extended the algorithm so that it can hypothesize a feature structure of a lexical entry by observing surrounding successful categories. As the algorithm works even for a complex feature like a subcategorization frame, it can be used to acquire a subcategorization dictionary. While some previous works on subcategorization frame acquisition assumed very little prior knowledge concerning the classification of subcategorization frames [Brent, 1991; Manning, 1993], our approach assumes the existence of grammar rules specifying subcategorization frame assignment, which enables more accurate learning of subcategorization frames.

Multiple Defects: In [Step 2] of the algorithm, it is supposed that each unsuccossfully parsed sentence has exactly onc cause of failure but a sentence in actual texts often contains two or more causes of failure (for example, two unknown words). To solve this problem, we extended the algorithm so that it searches for a multiple hypothesis which is a set of rewriting rulcs and lexical entries.

\section{Hypothesis Selection}

\subsection{Basic Grammatical Constraints}

From a linguistic point of view, hypotheses generated by the algorithm given above might contain many unnatural hypotheses because the algorithm itself does not have any linguistic knowledge to judge the appropriateness of hypotheses. 'To remove unnatural hypotheses, we have introduced the following criteria [Kiyono and T'sujii, 1993].

- T'he maximum number of adjacent unsuccessful categories is set to 2 in order not to decrease the efficiency of the algorithm.

- The maximum number of daughter nodes is set to 3 .

- Supposing that the existing grammar contains all the category conversion rules, a unary rule which has only one daughter node is not generated.
- Using generalizations embodied in the existing grammar, a hypothesis containing a sequence of subnodes which are collected into a larger category by existing grammar rules is not generated.

- Distinguishing non-lexical categories from lexical categories, a hypothesis whose mother category is a lexical category is not generated.

- Assuming that the existing grammar has a complete set of functional words, a lexical bypothesis is restricted to the open lexical categories, such as noun, verb, adjective, and adverb

\subsection{Constraint based on Local Boundaries}

A new constraint, on the violation of the boundary condition given to phrases was introduced to avoid any collection of adjacent successful categories in rule lypothesizing. Tho boundary condition is given by putting parentheses at both ends of a phrase, such as a noun phrase, a verb phrase, and a prepositional phrase. This constraint filters out a hypothesis which crosses cither end, not both ends, of a phrase. For example, when parentheses are put like "TThe default, blocking factor] is [20 blocks]", a hypothesis ' $V P \Rightarrow$ $V P, N P, V E R B B E$ " covering "blocking factor is" is discarded because of the violation of the boundary condition of a noun phrase "The default blocking factor"

This constraint requires the human task of putting parentheses before the hypothesis generator is invoked. In comparison with writing a constitume structure of the whole sentence, this work is much easier because we have only to give parentheses to definite phrases. Moreover, instcad of giving parentheses by liand, we can even obtain various tagged corpora.

As this constraint is also applicable to other constituents of the input sentence, it might improve the cfliciency of the top-down hypothesizing algorithm.

\subsection{Constraint based on X-bar Theory}

Most of the criteria in 3.1 are based on linguistic category classification but none of then cont mits itself to dealing with the relationship anong tho mother node and the daughter nodes. For example, supposing the existing grammar does not, contain a rule for participial adjuncts in nom phuases, the hypothesizing progran generates a new rewriting rule ' $N P \Rightarrow V P, N P$ ' from the plurase "blocking factor" in the sontence "l'he default blocking factor is 20 blocks". Ilowever, the program also gencrales olher alternative hypotheses from the same plirase, such as 'PI' $\Rightarrow V P, N P$ ' 'TNFTNITIVE' $\Rightarrow V P, N P$ ', and 'THAT'CI,AUSE' $\Rightarrow V P, N P$ ', each of which derives a post-positional adjunct for "default" by believing "default" is a head noun of the noun phrase. Linguistically, sucli combinations of mother nodes and danghter nodes are not allowed.

As a general principle for explaining phrase structures, $X$-bar theory is widely accepted. According to $\mathrm{X}$-bar theory, a grammar rule is (or can be converted to) either of the following forms, where cacls prime(') expresses the projection level of a head $X$. The projection level increases as grammar rules are applied and $X^{\prime \prime}$ is called a maximal projection of that category. $U$ and $W$ are adjuncts of $X^{\prime}$ and should be maximal projections of sone categories. 


$$
\begin{aligned}
& X^{\prime \prime} \Rightarrow Y X^{\prime} Z \\
& X^{\prime} \Rightarrow V X W
\end{aligned}
$$

If the existiug granumar is writen in $X$-bar leteory, this constraint is drastically eflective in reducing the? number of hypothesess.

\subsection{Plausibility of Hypotheses}

Anong the hypotheses which passed through all the constraints, each one hits a different plansibility as granmatical knowledge. Assuming that the existing grammar is reasonably comprelensive, lexical or idiosyneratic knowledge should be more platusible than general rewriting rules. In order to emphasize this ten. clency, anch hypollesis is given the following plausibil ity value.

$$
P\left(H y p o_{i}\right)=1-\frac{W\left(H y p o_{i}\right) \times H\left(H y o_{i}\right)}{W(S) \times H(S)}
$$

'This value is related to the proportion of the size, or the product of the width and the height, of the subthes composed by the hypothesis in the whole structure of the sentence. The value ranges from 0 to 1 and gets bigger if the hypothesis covers a smaller part of the sentence. The width of the hypothesis, $W\left(/ / y^{\prime} o_{i}\right)$, is delined as the word comt of the subtree and the height $I /\left(I y o_{i}\right)$ is as the shortest path liom lexical nodes to the lop node of the subtires.

\section{Experiments}

\subsection{Corpus}

In order to check the elfects of the hypothesis selection technicues, we carried ont some experiments with the UNIX on fine mannal. 100 semtences were chosen ats an experimental set from the manual. The characteristice of this corpus are as follows.

- Number of semtences: 100

- Jength of sentences: 9.08 words (average)

- Number of diflerent words: 381

- Hxamples:

There is no escape secquenee that prints a double puote.

Use the next argunent, as the blocking factor for tape records.

'I'le dedult, blocking factor is 20 blockss.

\subsection{Given Grammatical Knowledge}

'T'wo sets of grammar rules were prepared for the experiments, Grammar $A$ and Cirmment $B$. Crammar $A$ contaius 118 rewriting rules that cover basic expres. sions of linglish. (irannor $B$ is a subset of (irammat $A$ and contains only 25 rewriting rules. The contents of Grammar $A$ and Gammar $B$ are shown in 'Table 1.

The dictionary wo use is the fillo Huglish Dictio. nary containimg 200,000 entries. The entries of this alic lionary are not written in the form ol' a feature struce ture but have the enceded infermation of the syntactic patterns, which we interpret as a feature structure. $\Lambda$ s the WDR Dictionary was developed as a master dictionary for various applications, it took in the informa-

\begin{tabular}{|c|c|c|}
\hline Mother Category & Givamiar $A$ & Gratmonat is \\
\hline Sentence & 23 & 1 \\
\hline Vorb lirase & 40 & $1 \%$ \\
\hline Noun I'lirase? & 27 & $i$ \\
\hline Prepositional l'hrasse & 2 & 1 \\
\hline Adjective Phrase & 9 & 1 \\
\hline Aclverbial J'trase & 5 & 1 \\
\hline Infinitive Clantso & 4 & 1 \\
\hline 'I'lat clatuse & 1 & 1 \\
\hline Relative Clause & 6) & () \\
\hline Subordinale ('lauss & 1 & 0 \\
\hline Total & 118 & 25 \\
\hline
\end{tabular}
tion concerning all the appearances of each word with. out screening by frequencies. This chatactieristic of the
Table 1: Rule Conuts of 'l'wo Crammat Sots

FD)R Diclionary increases the andiguity of parsing. In fact, ach word within the sample sentences from the VNIX nanual has 1.49 parts of spects in the loOR Dictionary while the same vatue is 1.41 accordiug to the (OHIINS COBUHh) Hictionary.

\subsection{Generated Hypotheses}

Genemal Onteome: 'The experinents of gencrating hypotheses were carred out with Cirammar $A$ moder three diflerent conditions, (a) using the beasic grammatical constraints only, (b) adding the constrant with local pharasal boundaries given as parenthesess, and (c) adeling the constraint with $X$-bar theory. To carry out experiments (b) and (c), within the harget sentences, parentheses were given to noun phases, bufintive clauses, that-clauses, and subordinate clauses. A part of the result of experiment (a) is shown in 'Table 2 , each columm of which clisplays the number of hypotheses generated. 'L'te columms 'Single' and 'Mulliple" show the mumbers of single and multiple hypothesess respectively.

'The results of the there experiments are summarized in 'Table 3. The parser failed to analyse 61 ont of 100 seutences and the grantual hypothesizing progeam was invoked for those sentences. While no hypotheses were gencrated from $20 \mathrm{or} 30 \%$ of unsuccessfully parsed sentences becanse the current hypothesizing algerithm does not allow vertical duplicalion of incompleteness and also because the parancters of the basic grammatical constraints do mot allow the existence of more than t.wo adjacent incomplete nodes, the results on the numbers of actual hypotheses made show that the stronger 1he constraint we pose, the fower hypotheses are generated. The average hypotheses per sentence, calculated by dividing the total hypothesis combt of 1,301 in (a), $708 \mathrm{in}(\mathrm{b})$, and $231 \mathrm{im}(\mathrm{c})$, lyy the number of actual sentences from which hypotheses were generaled, 50 in (a), 14 in (b), and 41 in (c), was reduced from 26.0 to. 5.6 .

In sone cases, all the hypotheses are removed by newly introduced constraints, 6 sentences by the local houmlary constraint and 3 nore sentences by the constraint of $X$-bar theory. Investigation of the initial set of hypolleses genclated from such sentences revealod that no plansible hypothesis was included in it. 'Therefore, these sentences are not critical to the hypothesis selection method we introduced.

In the final set, of hypotheses, 30 phussible hypothe- 


\begin{tabular}{|c|c|c|c|c|c|c|}
\hline \multirow[t]{2}{*}{ Sentence } & \multicolumn{2}{|c|}{ Single } & \multicolumn{3}{|c|}{ Multiple } & \multirow[t]{2}{*}{ Total } \\
\hline & Lex & Trule & Tex & Mixed & Rule & \\
\hline The default blocking factor is 20 blocks. & 3 & 18 & 0 & 0 & $\overline{0}$ & 21 \\
\hline The output device in use is not capable of backspacing. & 4 & 26 & 0 & 0 & 0 & 30 \\
\hline Remove initial definitions for all predefined symbols. & 3 & 24 & 0 & 0 & () & 27 \\
\hline The escaped NEWLINE is not included in the macro value. & 0 & 0 & 2 & 2 & 0 & 4 \\
\hline Components of an expression are separated by white space. & 2 & 16 & 0 & 0 & 0 & 18 \\
\hline The name of this directory is listed in the folder variable. & 3 & 0 & 0 & 0 & 0 & 3 \\
\hline The name of the editor is listed in the EDITOR variable. & 2 & 0 & () & 0 & 0 & 2 \\
\hline
\end{tabular}

Table 2: Part of the Result of Experiment (a)

\begin{tabular}{|c|c|c|c|}
\hline & Experiment (a) & Txperiment (b) & Experiment (c) \\
\hline No. of Unsuccessfilly Parsed Sentences & 61 & 61 & 61 \\
\hline No. of Sentences which gencrated No Hypothesis & 11 & $\overline{17}$ & 20 \\
\hline No. of Sentences which generated Single Hypotheses & 43 & 39 & 37 \\
\hline No. of Sentences which generated Multiple IIypotheses & 7 & 5 & 4 \\
\hline No. of Sentences which generated Plausible Ilypotheses & 33 & 32 & 30 \\
\hline Rank of Plausible Hypotheses (Average) & 7.4 & 2.8 & 1.6 \\
\hline No. of Ilypotheses (Total) & 1301 & 708 & 231 \\
\hline No. of Hypotheses (Average) & 26.0 & 16.1 & 5.6 \\
\hline
\end{tabular}

Table 3: Hypotheses Generated from Different Conditions

ses, 7 new rewriting rules and 23 new or modified lexical entries, remained without being filtered out by newly introduced constraints. Some of the plausible hypotheses are listed below.

New Rule: $n p \Rightarrow$ np,adjp

New Rule: $n p \Rightarrow n p, n p$.

New Rule: $n p \Rightarrow v p, n p$. (from 3 sentences)

New Rule: np $\Rightarrow$ vppsv,np.

New Rule: $v p \Rightarrow v p, p$.

New Lexical Entry: $n \Rightarrow$ ['DELETE']

New Lexical Entry: $n \Rightarrow$ [pathrames]

Modified Lexical Entry: $v \Rightarrow$ [default]

Modified Lexical Entry: adj $\Rightarrow$ [invisible].

Modified Lexical Entry: adj $\Rightarrow$ [capable].

New Lexical Entry: adv $\Rightarrow$ [recursively].

......

The weighting function explained in 3.4 was not used for selecting hypotheses but the validity of it was proved by counting the order of cach plausible bypothesis in the set of generated hypotheses. The row of 'Rank of Plausible IIypotheses' in 'Table 3 indicates that plausible hypotheses stand much higher than the middle of the order

Examples: Ilereaftcr, in order to show how liypotheses were selected by each constraint, we explain the results for some typical examples.

Ex.1) "The default blocking factor is 20 blocks."

As Grammar $\Lambda$ does not contain a rule for participial adjuncts, the parser fails to analyse the noun phrase "the default blocking factor" and the granmar hypothesizing program is invoked. While this progran generates 21 liypotheses in experiment (a), it filters out the following 12 hypotheses in experinent (b). While checking local boundary violation, the program removes those grammatically unnatural combinations of categories, thongh it does not use any linguistic knowledge

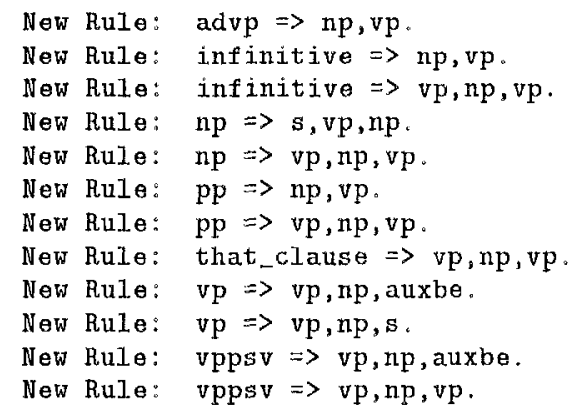

Moreover, the program filters out the following 1 hypothese with the constraint of X-bar theory.

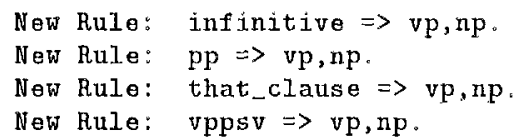

Finally, the following 5 hypotheses, anong which the expected hypothesis ' $N P$ ' $\Rightarrow V P, N P$ ' still remains are generated

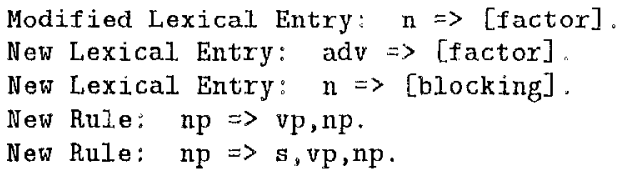

Ex.2) "The output device in use is not capable of backspacing."

This sentence is also parsed unsuccessfully because the current version of the PIDR Dictionary does not have information that "capable" subcategorizes a prepositional phrase. Among the initial set of 30 hypotheses, the following 8 hypotheses pass through 


\begin{tabular}{|l|r|r|}
\hline & Grammar A & Grammar B B \\
\hline No. of Unsucessfilly Parsed Sentences & 61 & 97 \\
\hline No. of Sentences which generated No Hypothesis & 11 & 45 \\
No. of Sentences which generatied Single Ilypotheses & 43 & 41 \\
No. of Sentences which generated Multiple llypotheses & 7 & 11 \\
\hline No. of Sentences wlich genorated Plausible Hypotheses & 31 & 16 \\
\hline \hline No. of IIypotheses (Thotal) & 1301 & 550 \\
No. of Iypotheses (Average) & 26.0 & 10.6 \\
\hline
\end{tabular}

Table 4: Ilypotheses Generaled from 'I'wo Granmar Sets

the constraints of local boundaries and X-bar theory. 'The first hypothesis in the list is the plausible hypothesis obtained in search of the real cause of the feature disagreement between "capable" and "ol backspacing". This lexical hypothesis for "capable" contains a inodified version of its subcategorization frante so that it subcategorizes of prepositional phrase.

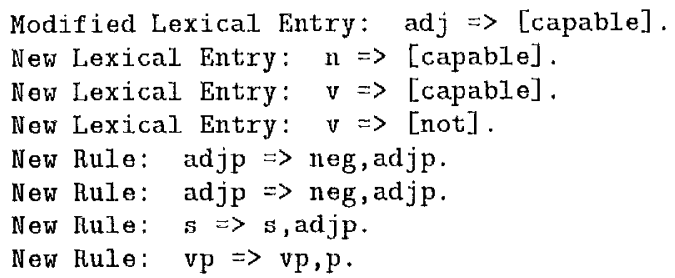

\subsection{Hypotheses from Smaller Knowledge}

Another experinent, was performed with (iranmate 13 under the hasic manmmatical constraints in order to compare the effects of the malurity of existing grammalical knowledge. The numbers of hypotheses generaled from two granmar sols are shown in Thable 4.

The coverage of Granmmar $B$ is so linited that 97 out of 100 sentences were parsed unsuecessfully and passed to the Ilypothesis Generator. However, as the immaturity of Grammar 13 also alfects the number of generated hypotheses, the number of plausible hypotheses anong the 550 hypotheses (10.6 hypotheses per sentence) generated from 97 sentences was only 16 . 'This result clame that cyclic accuisition of grammatical knowlodge is valid. liven the sentences from which no hypotheses are generated with a snall grammar would be taken into consideration in a later acquisition cycle with a larger grammar.

\section{Conclusion}

This paper proposed techuicutes for selecting appropriate lyypotheses in the rule-based processing stage of grammat accutisition. The experiments to eximne: the eflects of these techniques indicate that they have several advantages

- The newly introduced constraints reduce the number of hypotheses per sentence, from 20.0 to only 5.6, small enough to be treated in a compus-based processing environnent. This hypothesis selection is done without, discarding plansible hypotheses. Although, all the initial bypollieses may be, in certain cases, renoved by the new constraints, this happens only if uo plausible hypotlessis is included in the initial set.
- Even if no hypothesis is gencrated from an unsuccossfully parsed sentence (20 out of 61 sentences in experiment (c)) or no platusible hypothesis is inchuded in the initial hypothesis set (11 out of 41 sentences in experinent (c)), a plausible hypothesis will be renerated in the later acquisition cycte afler adding grammatical knowledge vilal for the senlence.

- Among the generated hypotheses, lexical hypotheses are nore plausible than rule hypotheses (23 out of 30 plausible hypotheses were lexical in experiment (c)). This fact means that the grammar used for the exporiments has an almost suflicient set of rewriting rules and that, after the grammar reaches such a mature situation during the acquisition cycle, only lexical or idiosyncratic knowledge has to be added. As our method has a facility to hypothesize a lexical entry with its leature structure including a subcategorization liame, we can set the larget of acquisition only to lexical knowledge for a large dictionary.

- 'The local boundary constraint was introduced for automatic hypothesis selection, but it might also be used in an interactive debugging tool for granmar maintenance.

\section{References}

[13rent, 1991] Michael R. 13rent. Antonatic Acquisition of Subcategorization Frames from Untagged 'Lext. In Proc of the $29 s i$ ACL meeting, pp.209 214,1991 .

[Jouglas and Dale, 1992] Shoma Douglas and Robert Dale. Towards Robust PAIK. In P'roc. of COLING92, рр.468 174, 1992 .

[(rocser, 1992] Sebastian Gocser. Clart Parsing of Robust Grammats. In Proc. of COLING-92, pp.120. 126,1992

[Kiyono and 'T'sujii, 1993] Masaki Kiyono and Jun'ichi 'l'sujii. Linguistic knowledge acquisition from parsing failures. In Proce of LAC CL, 93, p] .222 231, 1993.

[Manning, 1993] Christopher D. Manning. Automatic Acquisition of a Large Subcategorization Dictionary from Corpora. In Proce of the $31 \mathrm{st}$ ACL mecting, pp. $235-242,1993$.

[Matsumoto, 1986] Yuuji Malsumoto. A l'arallel l'arsing System for Natural Language Analysis. In lecture Notes in Computer Scicnce 225, SpringerVerlag, pp.394-409, 1986

[Mollish, 1989] ('hris S. Mellish. Some Chart-lansed Techniques for Parsing Ill-formed Input. In Proce. of the 2 \%th $A C$ C meeting, pp.102-109, 1989. 